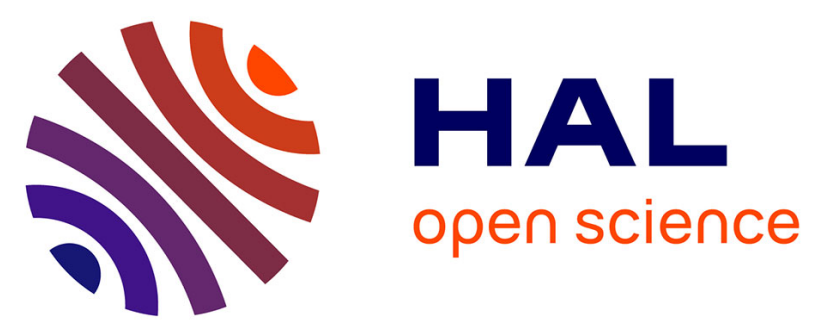

\title{
Location of intracranial aneurysms is the main factor associated with rupture in the ICAN population
}

Olivia Rousseau, Matilde Karakachoff, Alban Gaignard, Lise Bellanger, Philippe Bijlenga, Pacôme Constant Dit Beaufils, Vincent L'Allinec, Olivier Levrier, Pierre Aguettaz, Jean-Philippe Desilles, et al.

\section{To cite this version:}

Olivia Rousseau, Matilde Karakachoff, Alban Gaignard, Lise Bellanger, Philippe Bijlenga, et al.. Location of intracranial aneurysms is the main factor associated with rupture in the ICAN population. Journal of Neurology, Neurosurgery and Psychiatry, 2021, 92 (2), jnnp-2020-324371. 10.1136/jnnp2020-324371 . hal-03033899

\section{HAL Id: hal-03033899 https://hal.science/hal-03033899}

Submitted on 14 Oct 2021

HAL is a multi-disciplinary open access archive for the deposit and dissemination of scientific research documents, whether they are published or not. The documents may come from teaching and research institutions in France or abroad, or from public or private research centers.
L'archive ouverte pluridisciplinaire HAL, est destinée au dépôt et à la diffusion de documents scientifiques de niveau recherche, publiés ou non, émanant des établissements d'enseignement et de recherche français ou étrangers, des laboratoires publics ou privés. 
Title: Location of Intracranial Aneurysms is the Main Factor Associated with Rupture in the ICAN Population.

Cover title: Rupture and Intracranial Aneurysms Location

Olivia Rousseau, Msc; I Matilde Karakachoff, Msc; I Alban Gaignard, PhD; 2 Lise Bellanger, PhD; 3 Philippe Bijlenga, MD PhD; ${ }^{4}$ Pacôme Constant Dit Beaufils, Bsc; 5 Vincent L'Allinec, MD; 6 Olivier Levrier, MD PhD; 7 Pierre Aguettaz, MD; 7 Jean-Philippe Desilles, MD PhD; ${ }^{8}$ Caterina Michelozzi, MD; 9 Gaultier Marnat, MD; 10 Anne Clémence Vion, PhD; ${ }^{2}$ Gervaise Loirand, PhD; 2 Hubert Desal, MD PhD; "I Richard Redon, PhD; 2 Pierre-Antoine Gourraud, PhD MPH; ' Romain Bourcier, MD PhD; II on the behalf of the ICAN Investigators.

The ICAN Investigators are: Hubert Desal, Romain Bourcier, Richard Redon, Gervaise Loirand, JeanJacques Schott, Stéphanie Chatel, Emmanuelle Bourcereau, Christian Dina, Floriane Simonet, Eric Charpentier, Estelle Baron, Stéphanie Bonnaud, Benjamin Daumas-Duport, Bertrand Isidor, Jérôme Connault, Pierre Lebranchu, Antoine Rimbert, Thierry Le Tourneau, Chrisanthi Papagiannaki, Michel Piotin, Hocine Redjem, Mikael Mazighi, Jean Philippe Desilles, Olivier Naggara, Denis Trystram, Myriam Edjlali-Goujon, Grégoire Boulouis, Christine Rodriguez, Waghi Ben Hassen, Suzanna Saleme, Charbel Mounayer, Aymeric Rouchaud, Olivier Levrier, Pierre Aguettaz, Xavier Combaz, Anne Pasco, Vincent l'Allinec, Marc Bintner, Marc Molho, Pascale Gauthier, Cyril Chivot, Vincent Costalat, Cyril Darganzil, Alain Bonafé, Anne Christine Januel, Caterina Michelozzi, Christophe Cognard, Fabrice Bonneville, Philippe Tall, Jean Darcourt, Alessandra Biondi, Cristina losif, Jean Christophe Ferre, Jean Yves Gauvrit, François Eugene, Hélène Raoult, Jean Christophe Gentric, Julien Ognard, René Anxionnat, Benjamin Gory, Serge Bracard, Anne Laure Derelle, Romain Tonnelet, Laurent Spelle, Léon Ikka, Augustin Ozanne, Sophie Gallas, Jildaz Caroff, Nidal Ben Achour, Jacques Moret, Emmanuel Chabert, Jérôme 
Berge, Gaultier Marnat, Xavier Barreau, Florent Gariel, Frédéric Clarencon, Eimad Shotar, Mohammed Aggour, Frédéric Ricolfi, Adrien Chavent, Pierre Thouant, Pablo Lebidinsky, Brivael Lemogne, Denis Herbreteau, Richard Bibi, Kevin Janot, Laurent Pierot, Sébastien Soize, Marc Antoine Labeyrie, Christophe Vandendries, Emmanuel Houdart, Appoline Kazemi, Xavier Leclerc, Jean Pierre Pruvo, Nicolas Bricout, Stéphane Velasco, Samy Boucebci.

\section{Affiliations}

I University Hospital of Nantes, INSERM, CIC I4 I3, Clinique des données, F-44000 Nantes, France. 2 University of Nantes, CHU Nantes, CNRS, INSERM, L'institut du thorax, F-44000 Nantes, France.

3 University of Nantes, Laboratoire de Mathématiques Jean Leray UMR CNRS 6629, F-44000 Nantes, France.

4 Department of Clinical Neurosciences, Faculté de médecine, Université de Genève et Hôpitaux Universitaires de Genève, Switzerland.

5 Neurovascular Stroke unit, University Hospital of Nantes, INSERM, CIC I4I3, Clinique des données, F-44000 Nantes, France.

6 Department Neuroradiology, University Hospital of Angers, L'institut du thorax, INSERM I087, CNRS, UNIV Nantes, Nantes, France.

7 Hôpital Clairval-Ramsay GDS, Marseille, France.

8 Department of Interventional Neuroradiology, Rotschild Foundation hospital, Laboratory of Vascular Translational Science, UI I 48 INSERM, Université de Paris, Paris, France.

9 Department of Diagnostic and Therapeutic Neuroradiology, IRCCS Ospedale San Raffaele, Milan, Italy and Department of Diagnostic and Therapeutic Neuroradiology, CHU Toulouse, France.

10 Department of Diagnostic and Therapeutic Neuroradiology, CHU Bordeaux, France.

II Department of Diagnostic and Therapeutic Neuroradiology, University Hospital of Nantes, L'institut du thorax, Inserm 1087, CNRS, UNIV Nantes, Nantes, France.

Olivia Rousseau and Matilde Karakachoff contributed equally to this paper.

\section{Address correspondence to}

\section{Romain Bourcier}

Hôpital Laennec, Boulevard Professeur Jacques Monod, 44800 Saint-Herblain - France. Email: romain.bourcier@chu-nantes.fr 
olivia.rousseau@univ-nantes.fr, matilde.karakachoff@univ-nantes.fr, alban.gaignard@univ-nantes.fr, lise.bellanger@univ-nantes.fr, philippe.bijlenga@hcuge.ch, pacome.constantditbeaufils@chu-nantes.fr, vincent.lallinec@gmail.com, o-levrier@wanadoo.fr, aguettazpierre@gmail.com,

jpdesilles@gmail.com, caterina.michelozzi@gmail.com, gaultier.marnat@chu-bordeaux.fr, anneclemence.vion@univ-nantes.fr, gervaise.loirand@univ-nantes.fr, hubert.desal@chu-nantes.fr, richard.redon@univ-nantes.fr,.pierre-antoine.gourraud@univ-nantes.fr, romain.bourcier@chunantes.fr

Tables: 2

Figures: 4

Word count: 3110

Key Words: intracranial aneurysm, rupture, risks, location, machine learning, decision trees 


\section{ABSTRACT}

\section{Background and Purpose:}

The ever-growing availability of imaging led to increasing incidentally discovered unruptured intracranial aneurysms (UIAs). We leveraged machine-learning techniques and advanced statistical methods to provide new insights into rupture intracranial aneurysm (RIA) risks.

\section{Methods:}

We analysed characteristics of 2505 patients with intracranial aneurysms (IA) discovered between 2016 and 20I9. Baseline characteristics, familial history of IA, tobacco and alcohol consumption, pharmacological treatments before the IA diagnosis, cardiovascular risk factors and comorbidities, headaches, allergy and atopy, IA location, absolute IA size, and adjusted size ratio (aSR) were analysed with a multivariable logistic regression (MLR) model. A random forest (RF) method globally assessed the risk factors and evaluated the predictive capacity of a multivariate model.

\section{Results:}

Among 994 RIA (39.7\%) and I5I I UIA (60.3\%) patients, the MLR showed that IA location appeared to be the most significant factor associated to RIA (odds ratio (OR), 95\% confidence interval $(\mathrm{Cl})$ : internal carotid artery, reference; middle cerebral artery, 2.72, 2.02-3.58; anterior cerebral artery, 4.99, 3.6I-6.92; posterior circulation arteries, 6.05, 4.4I-8.33). Size and aSR were not significant factors associated with RIA in the MLR model, antiplatelettreatment intake patients were less likely to have RIA (OR: $0.74 ; 95 \% \mathrm{Cl}: 0.55-0.98)$. IA location, age, following by aSR were the best predictors of RIA using the RF model. 


\section{Conclusions:}

The location of IA is the most consistent parameter associated with RIA. The use of "artificial intelligence" RF helps to re-evaluate the contribution and selection of each risk factor in the multivariate model.

Key Words: intracranial aneurysm, rupture, risks, location, machine learning, decision trees 


\section{INTRODUCTION}

A ruptured intracranial aneurysm (RIA) is a vascular event with a mortality rate as high as $40 \%$.[I] It causes a loss of productive life-years similar to that of an ischemic stroke (the most common type of stroke), and its annual total economic burden in the United Kingdom was estimated to be GBP 510 million.[2] In the recent years, improved and more widely accessible non-invasive intracranial imaging techniques have led to an increased number of small, incidentally discovered unruptured IA (UIAs).[3,4] Generally, the overall prevalence of UIAs in the general population is estimated to be $3.2 \% .[5,6]$

Preventive treatment of UIA is a tangible management option given the treatment-related hazards as well as differential risk of rupture. In the absence of a randomized trial comparing treated patients with conservatively managed patients, treatment of UIAs remains both challenging and controversial, even if better outcomes have been reported for treated patients compared with conservatively managed patients.[7] Consequently, there are neither clear recommendations nor a consensus regarding the optimal management of UIA patients.[8,9] Past and current studies have suggested that UIAs may be classified as presenting a high or low rupture risk on the basis of their location and size.[10-14] Larger IAs and IAs in the posterior circulation arteries are thought to be related to a higher risk of RIAs. $[11,12,15]$ Furthermore, multiple IAs, [II] female sex,[I3] young age,[II,I2] history of RIA,[I6] and cigarette smoking have also been suggested as rupture-predisposing risk factors in various studies. Clinical decisions thus mainly rely on generic risk factors organized by prognostic scores, such as the PHASES (population, hypertension, age, size of aneurysm, earlier subarachnoid hemorrhage from another aneurysm, and site of aneurysm).[17] 
The Understanding the Pathophysiology of Intracranial Aneurysm project (ICAN) recruited a collection of patients with both RIAs or UIAs patients with extensive anatomical and epidemiological characterization as well as certified-expert clinical annotation. For instance, the ICAN project prospectively recorded data on tobacco consumption by pack-years, pharmacological treatments before the IA diagnosis, and very precise IA location for more than 20 variables in total.[18]

Machine-learning algorithms associated with large-scale computing infrastructure are currently accessible, and they have performed well in classification tasks such as patient stratification.[19] Leveraging both state-of-the-art machine-learning techniques and advanced statistical methods we provide new insights into IA rupture risks. 


\section{DATA AND METHODS}

\section{Data collection}

The ICAN project is an observational clinical research study approved by the Institutional Review Boards (Comité consultatif sur le traitement de l'information en matière de recherche dans le domaine de la santé, Commission Nationale de l'Informatique et des Libertés) and Ethics Committees of Nantes (GNEDS). Detailed information about the study's design, recruitment, and data collection has been previously published.[18] Patients recruited between May 2016 and May 2019 were included in the present study, accounting for 2742 individuals with a certified IA. Complete data were available for 2505 individuals (see Figure I Supplementary Material).

\section{Outcome and predictor variables}

The main outcome was a ruptured or unruptured IA. For patients with multiple aneurysms, the proponent IA was either the widest diameter one or the RIA itself. The variable "multiple IA" differentiates patients with a single IA from others. For RIA patient age is taken at rupture; for UIA patients, age is considered at inclusion. Data on sex, body mass index (BMI), and history of first-degree relative with IA are available in the ICAN study. Tobacco consumption was categorized based on the number of pack-years a smoker had consumed tobacco:[20] 0 for non-smokers, $>0$ to $<25$ for minor smokers, and 25 or more for regular smokers. Alcohol consumption was categorized by the amount of alcohol consumed per week: $\leq 150$ grams per week (g/week) and > $150 \mathrm{~g} /$ week. The study considers 27 variables, including (I) long termpharmacological treatments before the IA diagnosis (statins, platelet aggregation inhibiting, vitamin $\mathrm{K}$ antagonists (VKA) or anticoagulants, anti-inflammatories, and hormone therapies) 
and (2) medical history (ischemic stroke, ischemic heart disease, peripheral artery disease, carotid stenosis, aortic aneurysm, hypertension, headaches, diabetes, dyslipidemia, allergies, asthma, atopy, and eczema). Age and BMI, included as quantitative variables in the original data set, were transformed to qualitative variables in 4 classes (age: $<50,50-59,60-69, \geq 70$; BMI: $<20,20-25,25-30, \geq 30)$.

The IA locations were recorded according to the Trial on Endovascular Aneurysm Management (TEAM) scheme[2I] and then categorized as 4 classical groups: internal carotid artery (ICA), middle cerebral artery (MCA), anterior cerebral artery (ACA), and posterior circulation arteries (PCircA) (Figure II Supplementary Material).

The diameter of the RIA at time of rupture and of the UIA was measured in millimeters $(\mathrm{mm})$ using magnetic resonance imaging (MRI), computed tomography angiography (CTA), or digital subtraction angiography (DSA) and collected from reports by experts in charge of IA management in each center. The IA size was adjusted to consider the parent artery's diameter on the basis of the model of the size ratio proposed by Kashiwazaki.[22] (see Figure III Supplemental Material for details). Then, the adjusted size ratio (aSR) variable was transformed to a binary variable $(<3, \geq 3)$.

\section{Statistical analysis}

The factor analysis for mixed data (FAMD)[23] was applied to obtain a global description of data. This method detects groups of individuals and (or) variables and to locate outliers by using quantitative and qualitative variables together. This method can also provide a graphical display of all the individuals and the major sources of variance.

A chi-squared test, Student's t-test and a median test were applied to assess differences between ruptured and unruptured IA populations in baseline characteristics. Univariable 
logistic regression (LR) was applied to explore predictor variables in relation to rupture events. Then, a multivariable logistic regression (MLR) was applied in a two-step process. First, according to RIA literature,[17] sex, age, hypertension, tobacco consumption, IA location, aSR, and multiple IA variables were included in the model. Then, all significant variables of univariate LR were used, except for medical histories (ischemic stroke, heart disease and carotid artery stenosis history, dyslipidemia; excluded because these latter correlated to corresponding treatments). $P$-values and odds ratios (OR) with corresponding $95 \%$ confidence interval $(\mathrm{Cl})$ were calculated for both models.

Finally, a random forest (RF) method was applied to assess the predictive capacity of our model and reassess the MLR model. Training was applied to all variables contained in the dataset. Hyperparameters were tuned with the values 600 for the number of trees, 6 for the number of tested variables at each division, and 130 for the maximum number of nodes. The variables' importance was calculated through accuracy criteria.[24]

To evaluate the robustness of the MLR and RF models, a 10-fold cross-validation was performed. The maximum Youden index[25,26] was used to select the cutoff. Receiving operating characteristics (ROC) curves were used to measure and compare the performance of the two models implemented.

Data were analyzed with $R$ and Python statistical software. $R$ packages were used to compute the FAMD, MLR, and RF models (libraries FactoMineR, randomForest).[27,28] Python packages were used to clean data and to construct the baseline characteristics table. We implemented our data analysis through Jupyter Notebooks. Codes as well as simulated datasets are available at GitHub, for methods reuse and computational reproducibility (see Supplemental methods for complete details). 


\section{RESULTS}

Table I details the baseline characteristics of the 2505 patients according to the ruptured and unruptured status of the IA. Complete data were available for 2505 individuals, 994 (39.7\%) of whom had an RIA. The tobacco consumption in pack-years is significantly lower in the RIA group compared to the UIA group (I5.2 $\pm \mid 6.9$ versus $|6.8 \pm| 8.4, P=0.029)$.

In Figure I, the FAMD analysis was used to graphically show the 2505 included patients based on 27 variables over the 3 most important axes. The figure shows how ruptured and unruptured patients (red and blue circles) are intermingled and that no structure can be evidenced in the data concerning this feature. For explanatory purposes, we highlighted three patients (patients A, B, and C) whose features well represent each different axis characterization (the three patients' features are reported in Figure IV Supplementary Material). The overall ability to explain differences between RIA and UIA of the FAMD method reaches $20 \%$ cumulated over the 3 axes. The first axis ( $9.8 \%$ of explained variance) typically shows medical history and pharmacological treatments within particular dyslipidemia, statins, and platelet aggregation inhibiting treatments (patient A). The second axis $(6.3 \%$ of explained variance) is influenced by morphological IA characteristics (location and size) and sex (patient B). The third axis (5.2\% of explained variance) represents inflammatory components: atopy, allergy, and asthma (patient C) (The importance of the variables' contribution to the FAMD axes is shown in Figure V Supplementary Material).

Table 2 shows the importance of several previously identified variables. The patients' ages have an impact on IA rupture: older patients are less likely to have an RIA compared to younger ones (for age class, OR, and $P$ : $<50$, ref class; 50-59, 0.70, <0.00I; 60-69, 0.42, <0.00I; $\geq 70,0.28,<0.00 I)$. The IA location is the most associated factor with ruptured or unruptured status. The odds ratios decrease when passing from PCircA, to ACA, to MCA, and then to 
ICA. Moreover, the aSR also has an impact on IA rupture (OR 2.23; $95 \% \mathrm{Cl}$ : I.89-2.62, $P<0.00 \mathrm{I})$. Medical history variables also give indications for IA rupture: patients with an ischemic stroke history (OR 0.28; $95 \% \mathrm{Cl}: 0.19-0.4 \mathrm{I}, \mathrm{P}<0.00 \mathrm{I})$ and patients with an ischemic heart disease history (OR 0.26; $95 \% \mathrm{Cl}: 0.13-0.47, \mathrm{P}<0.00 \mathrm{I}$ ) are less likely to have an RIA.

Figure 2 shows the results of the MLR model (selection of variables is detailed in the Statistical analysis section). The IA location again appears to be the most significant factor of the MLR model: the odds ratio is more than 6 times higher for PCircA location compared to ICA (for IA location, OR, and 95\% Cl: ICA, reference; MCA, 2.72, 2.02-3.58; ACA, 4.99, 3.6I-6.92; PCircA, 6.05, 4.4I-8.33). Despite its significance in the LR model, the aSR is not significant in the MLR model (OR I.24; 95\% Cl: 0.97-I.57). The platelet aggregation inhibiting treatment is also a rupture discriminative factor: patients receiving this treatment appear to be less likely to have a ruptured IA (OR $0.74 ; 95 \% \mathrm{Cl}: 0.55-0.98)$ than others (the values used in the MLR model are reported in Table I Supplementary Material).

IA location, age, and aSR (adjusted IA size) are the 3 most important variables that have the best predictive power on the RF model (Figure 3). Ischemic stroke history and statin treatment also have a greater impact on the classification of ruptured and unruptured cases than other variables.

The ROC curves shown in figure 4 indicate similar performances in predicting rupture events using both the MLR and RF models. The area under the curve (AUC) is calculated at 0.74 (95\% Cl: $0.7 \mathrm{I}-0.76)$ for the MLR model and at 0.73 (95\% Cl: $0.7-0.75)$ for the RF model. 


\section{DISCUSSION}

In this analysis of a large nationwide and prospectively collected population of deeply phenotyped IA, the location of aneurysms is the most consistent parameter associated with RIA. In our study the IA location outweigh the importance of the IA size even though adjusted on parent artery in the prediction of rupture risk. Indeed, across different statistical analyses, we found an increasing number of RIAs along the gradient ICA, MCA, ACA, and PCircA.

The location of IA in the PCircA has already been associated with a higher risk of rupture. In the International Study of Unruptured Intracranial Aneurysms, the relative risk of rupture was higher for IA at the basilar tip and in the vertebrobasilar or posterior cerebral distribution compared with other locations.[14] A follow-up study confirmed that the RIA risk was higher for the same size categories involving aneurysms in the posterior circulation and posterior communicating artery compared with other locations.[7] Furthermore, as compared with IA in the MCAs, those in the posterior and anterior communicating arteries were more likely to rupture in the Japanese population of the Unruptured Cerebral Aneurysm Study during a follow-up period that included I I,660 aneurysm-years.[10]

We built a proxy (aSR) to consider the size ratio; we did this to consider the parent artery rather than characterizing only the absolute size of the IA. The size ratio was first described in 2013 in a study of 854 RIAs and I80 UIAs, which revealed that the size ratio, not the absolute size, may highly predict the risk of rupture in small UIAs.[29] In a systematic review and meta-analysis of 102 studies describing 144 risk factors, there was strong evidence for the morphological factors, including size ratio, to increase rupture risk.[30] The results of our univariate model indicated that an aSR $\geq 3$ is a factor associated with RIA. Despite the lack of significant results in the multivariate model, the RF model indicates that after the IA location, the aSR is one of the most informative variables in predicting ruptures. 
Considering the tools already available to predict the RIA, on the one hand the PHASES score attributes 3,6 , and 10 points to sizes above 7,10 , and $20 \mathrm{~mm}$, respectively, with the classical cut-off of $7 \mathrm{~mm}$ that is still often considered to be the leading parameter for treatment decisions. $[7,10]$ On the other hand, the PHASES score includes only 2 points for locations in the MCA and 4 points in the ACA and PCircA compared with 0 in the ICA (considered as the reference). Regarding IA characteristics, our study seems to indicate that the location of the IA could be a more prominent parameter to consider in the stratification of rupture risk.[17]

Statins and antiplatelet therapy are emphasized as important parameters regarding the risk of rupture in the RF and MLR models, respectively. Moreover, both variables appeared to characterize one of the dimensions evidenced in FAMD (first axis, 9.8\% of explained variance). Although recruitment criteria and selection characteristics of the study does not allow us to strongly conclude about protective and risk factors, both variables can be asserted as two important components of the IA population and associated to rupture. Several studies have highlighted the potential protective role of such therapy for the risk of RIA.[29] The supposed mechanisms involved their anti-inflammatory effects and their ability to stimulate the production of the extracellular matrix and the chemotactic migration of mesenchymal progenitor cells to stabilize the IA's parietal layers.[30] Interestingly, the largest case-control study to date, which involved 470 I patients with 64 I I IAs, showed that use of a lipid-lowering agent was significantly inversely associated with RIA.[3I] Subjects enrolled in the International Study of Unruptured Intracranial Aneurysms were compared regarding the frequency of aspirin use. The study revealed that patients taking aspirin 3 times weekly to daily experienced a significantly lower odds of RIA compared with those who never take aspirin.[32] The PROTECT-U (Prospective Randomized Open-label Trial to Evaluate risk faCTor management 
in patients with UIA that randomize medical strategy to reduces the risk of RIA) study may provide more insights into these prospects.[33]

Surprisingly, an older age seems to be associated with UIAs in the MLR analysis. Indeed, an older age is known to increase the risk of an RIA.[7, 17] One potential explanation for this finding is that age and smoking history are potentially associated with cardiovascular comorbidities (history of ischemic stroke or heart disease) and reflect a global selection bias. Indeed, in the older and diseased population, there is a higher chance of discovering incidental UIAs because of cerebral vessel imaging performed during general work-ups. The smoking habit is one of the main risk factors in both the presence and rupture of an $I A,[6, I 2, I 7]$ and it is unlikely that, as with ischemic heart disease, a smoking paradox was involved in the IA epidemiology.[34] As previously reported, in this population exposed to cardiovascular comorbidities, easier access to imaging may explain an increased proportion of incidentally discovered UIAs.[35]

Hypertension is a classically reported risk factor for RIAs in epidemiological studies.[17,36] In our study, no significant association was found between hypertension and rupture. Indeed, UIA patients, presenting significantly more cardiovascular comorbidities than RIA patients, are probably thoroughly examined and needs to have strictly controlled blood pressure in secondary prevention.[37]

Headaches history was found as inversely associated with RIA. However, headache is one of the symptoms that lead to imaging screening and UIAs incidental discovery, as reported in previous studies.[7,37,38]

Our study has many strengths. It includes data from a large nationwide and prospectively collected population of well-phenotyped IAs. Patients were recruited from 35 different centers across all the country, at the same period, following the same protocol and same 
standardized questionnaires. Our population of 2505 patients with IAs was $71.9 \%$ female and had a median age of $53.6( \pm \mid 2.3)$, which compares well with the large IA populations previously studied.[7, I0, I4, I7] Furthermore, comparison of principal characteristics of UIA patients across different international studies highlights similarities between populations.[7,10] (Table III in Supplementary Material).

We acknowledge that there are limitations in our study that are mainly due to its design, resulting in previously mentioned selection bias. First, as already hypothesized in other studies,[37,38] UIA patients are potentially recruited after a fortuitous IA discovery during an imaging work-up for ischemic stroke or ischemic heart disease. Moreover, the uncertainty of temporal sequences of RIAs makes our analysis incomparable and we have no intention to challenge longitudinal studies, such as those that were used to build the PHASES scoring system. Second, we only included non-Finnish Europeans in our database because the ICAN is a nationwide project. Third, data about the parent artery diameter was absent at the time of database extraction; therefore, we only used a proxy by applying a ratio to the IA location. However, thanks to the TEAM trial scheme, which helped determine an accurate location, we intend to limit the variability of that approximation. Finally, data from other features such as medical imaging and genetic variations are lacking but are currently under active investigation and may be combined with clinical features.

Overall, our study highlights the importance of the morphological and anatomic factors of the IA compared to the environmental risk factors. We confirm the importance of well-known parameters in evaluating the risk of an RIA, such as the size and the location of IA. Furthermore, because of the complementary and advanced statistical methods implemented here (MLR and RF), we are able to refine the relative importance of size and location and thus emphasize a probably underestimated importance of the IA location over IA size. Finally, we 
make a new epidemiological argument for the promising protective role of oral medication. which could stabilize an IA's parietal layers.

\section{CONCLUSIONS}

The location of an IA is the most consistent parameter associated with an RIA. This study includes only typical bifurcation IAs; however, imaging data may be soon available for each patient, so we may be able to add numerous quantitative parameters describing the anatomical and flow patterns to our predictive model.

\section{DISCLOSURES}

None 


\section{REFERENCES}

I Nieuwkamp DJ, Setz LE, Algra A, et al. Changes in case fatality of aneurysmal subarachnoid haemorrhage over time, according to age, sex, and region: a meta-analysis. Lancet Neurol 2009;8:635-42. doi:10.1016/SI474-4422(09)70I26-7

2 Rivero-Arias O, Gray A, Wolstenholme J. Burden of disease and costs of aneurysmal subarachnoid haemorrhage (aSAH) in the United Kingdom. Cost Eff Resour Alloc 2010;8:6. doi: I0.1 I86/1478-7547-8-6

3 Bijlenga $P$, Ebeling $C$, Jaegersberg $M$, et al. Risk of rupture of small anterior communicating artery aneurysms is similar to posterior circulation aneurysms. Stroke 20I3;44:30 I8-26. doi:I0.II6I/STROKEAHA.II3.00I667

4 Vernooij MW, Ikram MA, Tanghe HL, et al. Incidental findings on brain MRI in the general population. N Engl J Med 2007;357:I82 I-8. doi:I0.I056/NEJMoa070972

5 Rinkel GJE, Djibuti M, Algra A, et al. Prevalence and risk of rupture of intracranial aneurysms: a systematic review. Stroke 1998;29:25I-6. doi:I0.II6I/0I.STR.29.I.25 I

6 Vlak MH, Algra A, Brandenburg R, et al. Prevalence of unruptured intracranial aneurysms, with emphasis on sex, age, comorbidity, country, and time period: a systematic review and meta-analysis. Lancet Neurol 20I I;I 0:626-36. doi:I0.10 I6/SI4744422(II)70109-0

7 Wiebers DO. Unruptured intracranial aneurysms: natural history, clinical outcome, and risks of surgical and endovascular treatment. Lancet 2003;362:103-10. doi:I0.10I6/S0|40-6736(03) I3860-3

8 Naggara O, Darsaut T, Trystram D, et al. Unruptured intracranial aneurysms: why we must not perpetuate the impasse for another 25 years. Lancet Neurol 20 I 4; I 3:537-8. doi: $10.1016 / S 1474-4422(14) 70091-2$

9 Naggara ON, Lecler A, Oppenheim C, et al. Endovascular treatment of intracranial unruptured aneurysms: a systematic review of the literature on safety with emphasis on subgroup analyses. Radiology 20 I2;263:828-35. doi: I0. I |48/radiol. I2 I I 2 | |4

10 The UCAS Japan Investigators. The natural course of unruptured cerebral aneurysms in a Japanese cohort. N Engl J Med 20 I2;366:2474-82. doi:I0. I056/NEJMoa I I I 3260

I I Sonobe M, Yamazaki T, Yonekura M, et al. Small unruptured intracranial aneurysm verification study: SUAVe study, Japan. Stroke 2010;4 I:1969-77.

doi:I0.I I6I/STROKEAHA.I 10.585059

12 Juvela S. Natural history of unruptured intracranial aneurysms: risks for aneurysm formation, growth, and aupture. Acta Neurochir Suppl 2002;82:27-30. doi:10.1007/978-3709|-6736-6_5

13 Wermer $\mathrm{MJH}$, van der Schaaf IC, Algra A, et al. Risk of rupture of unruptured intracranial aneurysms in relation to patient and aneurysm characteristics: an updated meta-analysis. Stroke 2007;38:I404-10. doi:I0.I I6I/0 I.STR.0000260955.5 I40 I.cd 
14 Unruptured intracranial aneurysms - risk of rupture and risks of surgical intervention. N Engl J Med 1998;339:1725-33. doi:I0.1056/NEJMI998I210339240 I

I5 Molyneux AJ, Kerr RS, Yu L-M, et al. International subarachnoid aneurysm trial (ISAT) of neurosurgical clipping versus endovascular coiling in 2143 patients with ruptured intracranial aneurysms: a randomised comparison of effects on survival, dependency, seizures, rebleeding, subgroups, and aneurysm occlusion. Lancet 2005;366:809-I7. doi:I0.10I6/S0I40-6736(05)672/4-5

16 Ishibashi T, Murayama Y, Urashima M, et al. Unruptured intracranial aneurysms: incidence of rupture and risk factors. Stroke 2009;40:3 I3-6.

doi:I0.1 I6I/STROKEAHA.I08.52I674

17 Greving JP, Wermer MJH, Brown RD, et al. Development of the PHASES score for prediction of risk of rupture of intracranial aneurysms: a pooled analysis of six prospective cohort studies. Lancet Neurol 2014;1 3:59-66. doi:I0.1016/SI4744422(I3)70263-I

I8 Bourcier R, Chatel S, Bourcereau E, et al. Understanding the pathophysiology of intracranial aneurysm: the ICAN project. Neurosurgery 2017;80:62I-6.

doi:I0.1093/neuros/nywl35

19 Rajkomar A, Dean J, Kohane I. Machine learning in medicine. $N$ Engl J Med 2019;380:I347-58. doi:I0.I056/NEJMra|8|4259

20 Leffondre K. Modeling smoking history: a comparison of different approaches. Am J Epidemiol 2002; I 56:8I3-23. doi: I0.1093/aje/kwfl 22

2I Raymond J, Chagnon M, Collet J-P, et al. A randomized trial on the safety and efficacy of endovascular treatment of unruptured intracranial aneurysms is feasible. Interv Neuroradiol 2004; 1 0:103-12. doi:10.1 I77/159101990401000202

22 Kashiwazaki D, Kuroda S, on behalf of the Sapporo SAH Study Group. Size ratio can highly predict rupture risk in intracranial small $(<5 \mathrm{~mm})$ aneurysms. Stroke 2013;44:2169-73. doi:10.1 I6I/STROKEAHA. I I3.001 I 38

23 Pagès J. Analyse factorielle de données mixtes. Rev Stat Appl 2004;52:93-IIII.

24 Strobl C, Boulesteix A-L, Zeileis A, et al. Bias in random forest variable importance measures: illustrations, sources and a solution. BMC Bioinformatics 2007;8:25. doi: 10.1 | 86/|47|-2105-8-25

25 Youden WJ. Index for rating diagnostic tests. Cancer 1950;3:32-5. doi:10.1002/1097$0 \mid 42$ (I950)3: I<32::aid-cncr2820030106>3.0.co;2-3

26 Perkins NJ, Schisterman EF. The Youden index and the optimal cut-point corrected for measurement error. Biom J 2005;47:428-4I. doi:10.1002/bimj.2004I0I33

27 Lê S, Josse J, Husson F. FactoMineR: an R package for multivariate analysis. J Stat Soft 2008;25. doi:10.18637/jss.v025.i0 I

28 Breiman L. Random forest. Mach Learn 200I;45:5-32. doi:I0.1023/A:1010933404324 
29 Aoki T. Future prospects for the development of a novel medical therapy for intracranial aneurysm. Folia Pharmacol Jap 2016; I 48:86-9I. doi: I0.1254/fpj. I 48.86

30 Brinjikji W, Yong Hong D, Dai D, et al. Statins are not associated with short-term improved aneurysm healing in a rabbit model of unruptured aneurysms. J Neurointerv Surg 2017;9:200-3. doi:10.1 I36/neurintsurg-2016-012265

3I Can A, Castro VM, Dligach D, et al. Lipid-lowering agents and high HDL (high-density lipoprotein) are inversely associated with intracranial aneurysm rupture. Stroke 2018;49: I|48-54. doi:I0.1 I6I/STROKEAHA. I I7.019972

32 Hasan DM, Mahaney KB, Brown RD, et al. Aspirin as a promising agent for decreasing incidence of cerebral aneurysm rupture. Stroke 201 I;42:3156-62.

doi:|0.| I6I/STROKEAHA. I I I.6I94II

33 Vergouwen MD, Rinkel G], Algra A, et al. Prospective randomized open-label trial to evaluate risk factor management in patients with unruptured intracranial aneurysms: study protocol. Int J Stroke 20 I8; I 3:992-8. doi: I 0.I I 77/I 7474930 I8790033

34 Aune E, Røislien J, Mathisen M, et al. The "smoker's paradox" in patients with acute coronary syndrome: a systematic review. BMC Med 20 I 1;9:97. doi:I0. I I86/I74 I-70I59-97

35 Rinkel GJE. Management of patients with unruptured intracranial aneurysms: Curr Opin Neurol 2019;32:49-53. doi:I0.1097/WCO.0000000000000642

36 Lindgren AE, Kurki MI, Riihinen A, et al. Hypertension predisposes to the formation of saccular intracranial aneurysms in 467 unruptured and 1053 ruptured patients in Eastern Finland. Ann Med 2014;46: I69-76. doi: 10.3 I09/07853890.20I4.883 I68

37 Vlak MHM, Rinkel GJE, Greebe P, et al. Risk of rupture of an intracranial aneurysm based on patient characteristics: a case-control study. Stroke 2013;44:1256-9. doi:I0.1 I6 I/STROKEAHA. I I I.000679

38 Hostettler IC, Alg VS, Shahi N, et al. Characteristics of unruptured compared to ruptured intracranial aneurysms: a multicenter case-control study. Neurosurgery 2018;83:43-52. doi:10.1093/neuros/nyx365 


\section{FIGURES LEGENDS}

Figure I. Graphical display of the FAMD presenting the global profiles of the ICAN study patients. Patients with a ruptured IAs (red) or an unruptured IAs (blue) are represented on this graph. The position relative to each axis gives some patients' characteristics. Also shown are the locations of 3 patients who are used as examples (Figure II Supplementary Material) and confirm axis characterization. Each axis corresponds to a principal component of FAMD method. The percentage of variance explained by each axis is given in parenthesis.

Abbreviations: $A C A=$ Anterior cerebral artery; FAMD = Factor analysis for mixed data; $I A$ $=$ Intracranial aneurysm

Figure 2. Forest plot of a multivariable logistic regression to explain IA rupture. The model's variables are represented depending on their labels. A visual representation of the odds ratio is given in addition to their values.

Abbreviations: $\mathrm{ACA}=$ Anterior cerebral artery; $\mathrm{Cl}=$ Confidence interval; $\mathrm{HTA}=$ Hypertension; IA = Intracranial aneurysm; ICA = Internal carotid artery; MCA = Middle cerebral artery; $\mathrm{PCircA}=$ Posterior circulation arteries

Figure 3. Variables' importance in random forest model. After each variable name, the number of labels is indicated in the parenthesis. Variables are classified by most important to less important impact in the random forest model.

Abbreviations: $\mathrm{IA}=$ Intracranial aneurysm; $\mathrm{PAD}=$ Peripheral artery disease; $\mathrm{VKA}=$ Vitamin $\mathrm{K}$ antagonist 
Figure 4. ROC curves comparison. The performance of this method is evaluated with a I0-fold cross-validation. In the legend, the AUC for each method is given by mean and standard deviation interval. ROC curves are intersected several times.

Abbreviations: $A \cup C=$ Area under the curve; $R O C=$ Receiver operating characteristic 


\section{TABLES}

Table I. Baseline characteristics of the ICAN study and subgroups used to compare ruptured and unruptured IA patients.

\begin{tabular}{|c|c|c|c|c|}
\hline Characteristic & All $(n=2505)$ & RIA $(n=994)$ & UIA $(n=|5| I)$ & P-value \\
\hline Sex (women) & I80I (7I.9\%) & $692(69.6 \%)$ & I I 09 (73.4\%) & 0.044 \\
\hline Age (years) & $53.6( \pm 12.3)$ & $50.3( \pm 12.1)$ & $55.8( \pm 11.9)$ & $<0.001$ \\
\hline Body mass index & $25.4( \pm 4.8)$ & $25.4( \pm 4.8)$ & $25.4( \pm 4.8)$ & 0.744 \\
\hline IA familial history (yes) & $454(18.1 \%)$ & $162(16.3 \%)$ & $292(19.3 \%)$ & 0.061 \\
\hline \multicolumn{5}{|c|}{ IA characteristics } \\
\hline IA size $(\mathrm{mm})$ & $6.0[4.2-8.0]$ & $6.0[4.0-8.0]$ & $6.0[4.4-8.0]$ & 0.039 \\
\hline Adjusted Size Ratio & $3.0[1.75-5.0]$ & $3.5[2.0-5.7]$ & $2.4[1.5-5.0]$ & $<0.001$ \\
\hline IA location & $622(24.8 \%)$ & 117 (II.8\%) & $505(33.4 \%)$ & $<0.001$ \\
\hline MCA & $662(26.4 \%)$ & $231(23.2 \%)$ & $431(28.5 \%)$ & 0.004 \\
\hline ACA & 745 (29.7\%) & $383(38.5 \%)$ & $362(24.0 \%)$ & $<0.001$ \\
\hline PCircA & $476(19.0 \%)$ & $263(26.5 \%)$ & $213(14.1 \%)$ & $<0.001$ \\
\hline Multiple IA (yes) & 796 (31.8\%) & $338(34.0 \%)$ & $458(30.3 \%)$ & 0.058 \\
\hline \multicolumn{5}{|c|}{ Medical history } \\
\hline Hypertension (yes) & 931 (37.2\%) & $362(36.4 \%)$ & 569 (37.7\%) & 0.558 \\
\hline Headaches (yes) & 427 (17.0\%) & | 46 (|4.7\%) & $281(18.6 \%)$ & 0.013 \\
\hline
\end{tabular}




\begin{tabular}{|c|c|c|c|c|}
\hline Dyslipidemia (yes) & $570(22.8 \%)$ & 186 (I8.7\%) & $384(25.4 \%)$ & $<0.001$ \\
\hline $\begin{array}{c}\text { Ischemic stroke history } \\
\text { (yes) }\end{array}$ & $187(7.5 \%)$ & $31(3.1 \%)$ & $156(10.3 \%)$ & $<0.001$ \\
\hline $\begin{array}{c}\text { Ischemic heart disease } \\
\text { history (yes) }\end{array}$ & $79(3.2 \%)$ & $12(1.2 \%)$ & 67 (4.4\%) & $<0.001$ \\
\hline Statin treatment (yes) & 379 (15.1\%) & I I2 (I I.3\%) & 267 (17.7\%) & $<0.001$ \\
\hline $\begin{array}{c}\text { Platelet aggregation } \\
\text { inhibiting treatment } \\
\text { (yes) }\end{array}$ & $343(13.7 \%)$ & 98 (9.9\%) & $245(16.2 \%)$ & $<0.001$ \\
\hline $\begin{array}{l}\text { Hormone therapy } \\
\text { treatment (yes) }\end{array}$ & $78(3.1 \%)$ & $22(2.2 \%)$ & $56(3.7 \%)$ & 0.047 \\
\hline Allergy (yes) & $584(23.3 \%)$ & $202(20.3 \%)$ & $382(25.3 \%)$ & 0.005 \\
\hline \multicolumn{5}{|c|}{ Habits } \\
\hline Tobacco Non-smoker & 731 (29.2\%) & $289(29.1 \%)$ & $442(29.3 \%)$ & 0.959 \\
\hline Minor smoker & 1090 (43.5\%) & 455 (45.8\%) & $635(42.0 \%)$ & 0.070 \\
\hline Regular smoker & $684(27.3 \%)$ & $250(25.2 \%)$ & $434(28.7 \%)$ & 0.055 \\
\hline Tobacco (pack-years) & $16.1( \pm 17.8)$ & $15.2( \pm 16.9)$ & $16.8( \pm 18.4)$ & 0.029 \\
\hline Alcohol (> I50 g) & 289 (II.5\%) & $128(12.9 \%)$ & 161 (10.7\%) & 0.101 \\
\hline
\end{tabular}

Values are expressed as numbers (\%) for categorical variables and mean ( \pm standard deviation) or median [first quartile-third quartile] for quantitative variables depending on their distribution (normal vs skewed). Student's t-test and $\chi^{2}$ test were applied to see if there is a difference between patients with a ruptured or an unruptured IA. 
Abbreviations: $A C A=$ Anterior cerebral artery; $g$ = grams; $I A=$ Intracranial aneurysm; $I C A=$ Internal carotid artery; $\mathrm{MCA}=$ Middle cerebral artery; $\mathrm{mm}=$ millimeters; PCircA= Posterior circulation arteries

Definitions:

Adjusted IA size= the IA size is modified depending on the IA location to compare IA on arteries of approximately the same diameter.

Pack-years $=$ Calculation made by multiplying the smoking time (years) by the number of cigarettes consumed per day. 
Table 2. Odds ratio calculation for univariable logistic regression to explain IA rupture.

\begin{tabular}{|c|c|}
\hline Explanatory variables & OR (univariate) \\
\hline Sex (Male) & $1.20(1.01-1.44, P=0.040)$ \\
\hline Age $(<50)$ & $0.70(0.58-0.85, P<0.00 I)$ \\
\hline $60-69$ & $0.42(0.34-0.52, P<0.00 I)$ \\
\hline$\geq 70$ & $0.28(0.20-0.38, P<0.00 I)$ \\
\hline BMI $\left(\mathrm{kg} / \mathrm{m}^{2}\right)$ & $1.00(0.98-1.01, P=0.744)$ \\
\hline Sporadic case (Yes) & $1.23(1.00-1.52, P=0.055)$ \\
\hline Multiple IA (Yes) & $1.18(1.00-1.41, P=0.052)$ \\
\hline IA location (ICA) & $2.3 I(I .79-3.00, P<0.00 I)$ \\
\hline $\mathrm{ACA}$ & $4.57(3.58-5.86, P<0.00 I)$ \\
\hline PCircA & $5.33(4.08-7.00, P<0.00 I)$ \\
\hline Adjusted size ratio $(\geq 3)$ & $2.23(I .89-2.62, P<0.00 I)$ \\
\hline Tobacco (Non-smoker) & $1.10(0.91-1.33, P=0.347)$ \\
\hline Regular smoker & $0.88(0.7 \mathrm{I}-1.09, P=0.248)$ \\
\hline Alcohol (> $150 \mathrm{~g})$ & $1.24(0.97-1.59, P=0.089)$ \\
\hline Hypertension (Yes) & $0.95(0.80-1.12, P=0.530)$ \\
\hline Headaches (Yes) & $0.75(0.60-0.94, P=0.01 \mathrm{I})$ \\
\hline Diabetes (Yes) & $0.69(0.46-1.02, P=0.069)$ \\
\hline Dyslipidemia (Yes) & $0.68(0.55-0.82, P<0.00 I)$ \\
\hline Ischemic stroke history (Yes) & $0.28(0.19-0.4 I, P<0.00 I)$ \\
\hline Ischemic heart disease history (Yes) & $0.26(0.13-0.47, P<0.00 I)$ \\
\hline PAD history (Yes) & $0.69(0.38-1.22, P=0.216)$ \\
\hline Carotid artery stenosis history (Yes) & $0.50(0.28-0.85, P=0.014)$ \\
\hline Aortic aneurysm history (Yes) & $0.5 I(0.1 \mid-I .70, P=0.307)$ \\
\hline Statin treatment (Yes) & $0.59(0.47-0.75, P<0.00 I)$ \\
\hline Platelet aggregation inhibiting treatment (Yes) & $0.57(0.44-0.72, P<0.00 I)$ \\
\hline
\end{tabular}




\begin{tabular}{|l|l|}
\hline VKA or anticoagulant treatment (Yes) & $0.72(0.43-1.17, P=0.198)$ \\
\hline Anti-inflammatory treatment (Yes) & $0.83(0.52-1.30, P=0.417)$ \\
\hline Hormone therapy treatment (Yes) & $0.59(0.35-0.96, P=0.037)$ \\
\hline Allergy (Yes) & $0.75(0.62-0.91, P=0.004)$ \\
\hline Asthma (Yes) & $0.82(0.59-1.13, P=0.227)$ \\
\hline Atopy (Yes) & $0.88(0.6 \mathrm{I}-1.26, P=0.492)$ \\
\hline Eczema (Yes) & $0.96(0.68-1.34, P=0.793)$ \\
\hline
\end{tabular}

Values are expressed as odds ratio ( $95 \%$ confidence interval, $\mathrm{p}$-value).

Abbreviations: $\mathrm{BMI}=$ Body mass index; $\mathrm{IA}=$ intracranial aneurysm; ICA $=$ Internal carotid artery; $\mathrm{kg} / \mathrm{m}^{2}=$ kilograms per square meter; $\mathrm{MCA}=$ Middle cerebral artery; $\mathrm{ACA}=$ Anterior cerebral artery; OR = Odds ratio; PCircA = Posterior circulation arteries; PAD = Peripheral artery disease; $\mathrm{VKA}=$ Vitamin $\mathrm{K}$ antagonist 


\section{FIGURES}

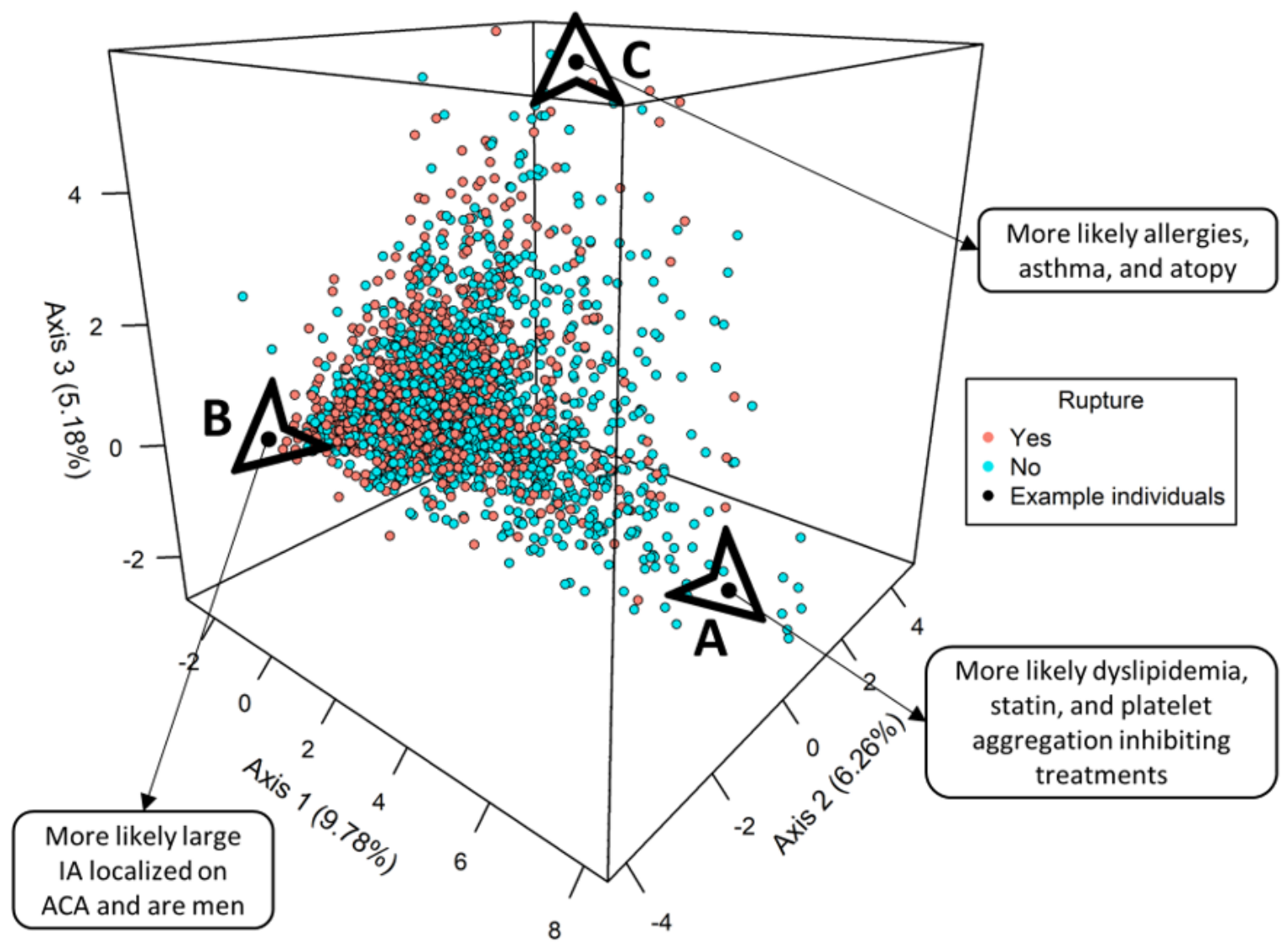

Figure I. Graphical display of the FAMD presenting the global profiles of the ICAN study patients. 


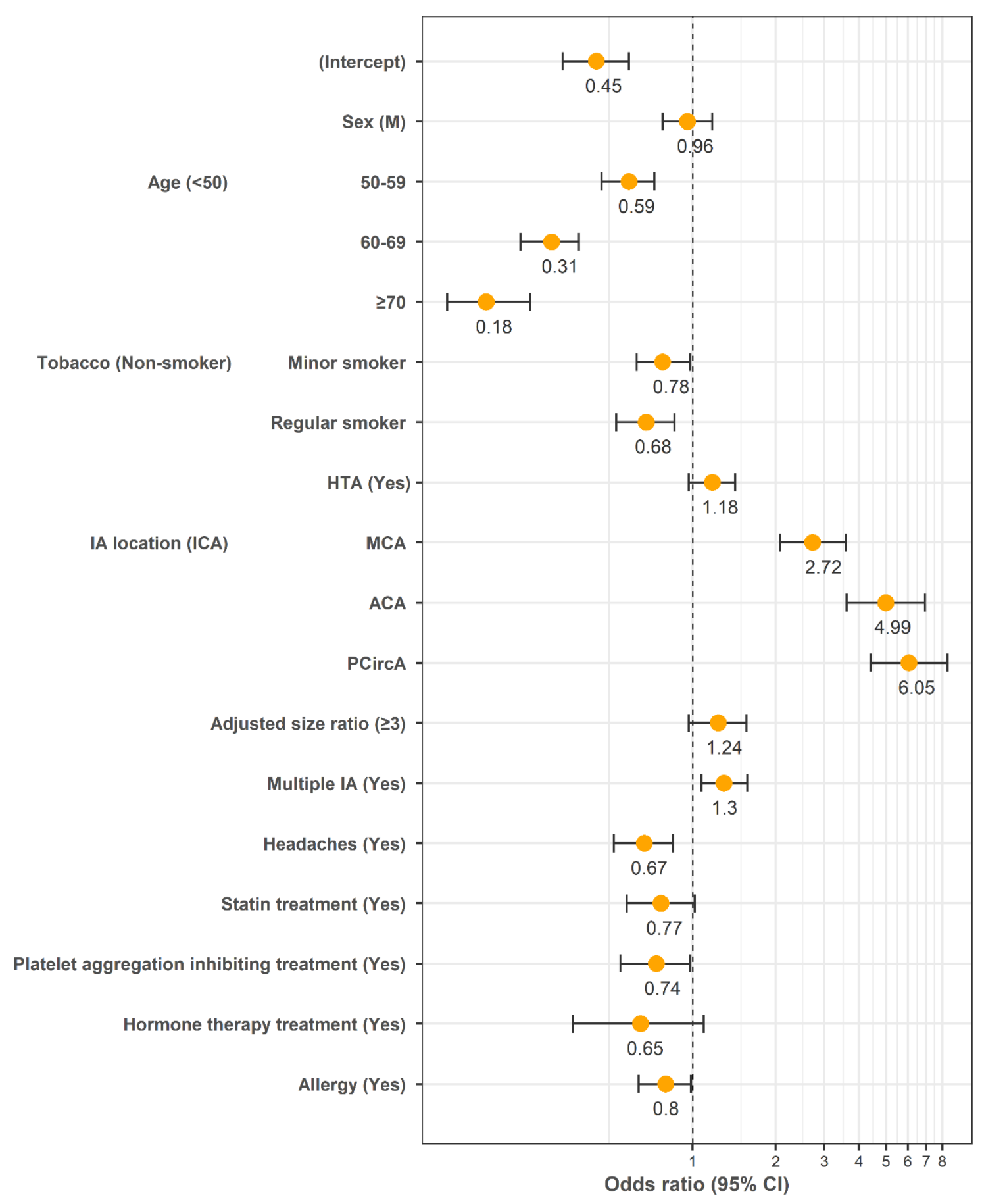

Figure 2. Forest plot of a multivariable logistic regression to explain IA rupture. 


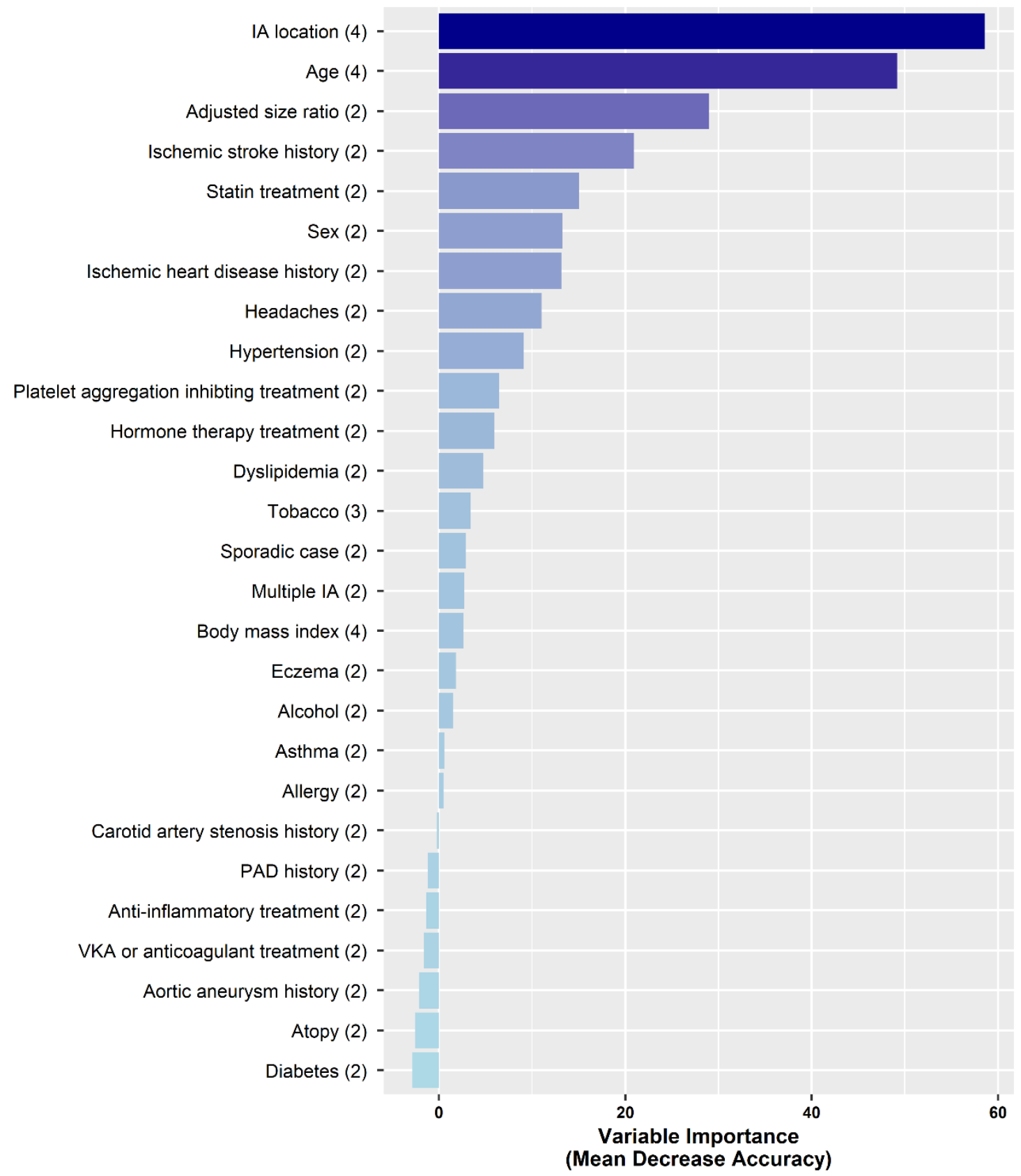

Figure 3. Variables importance in Random Forest model. 


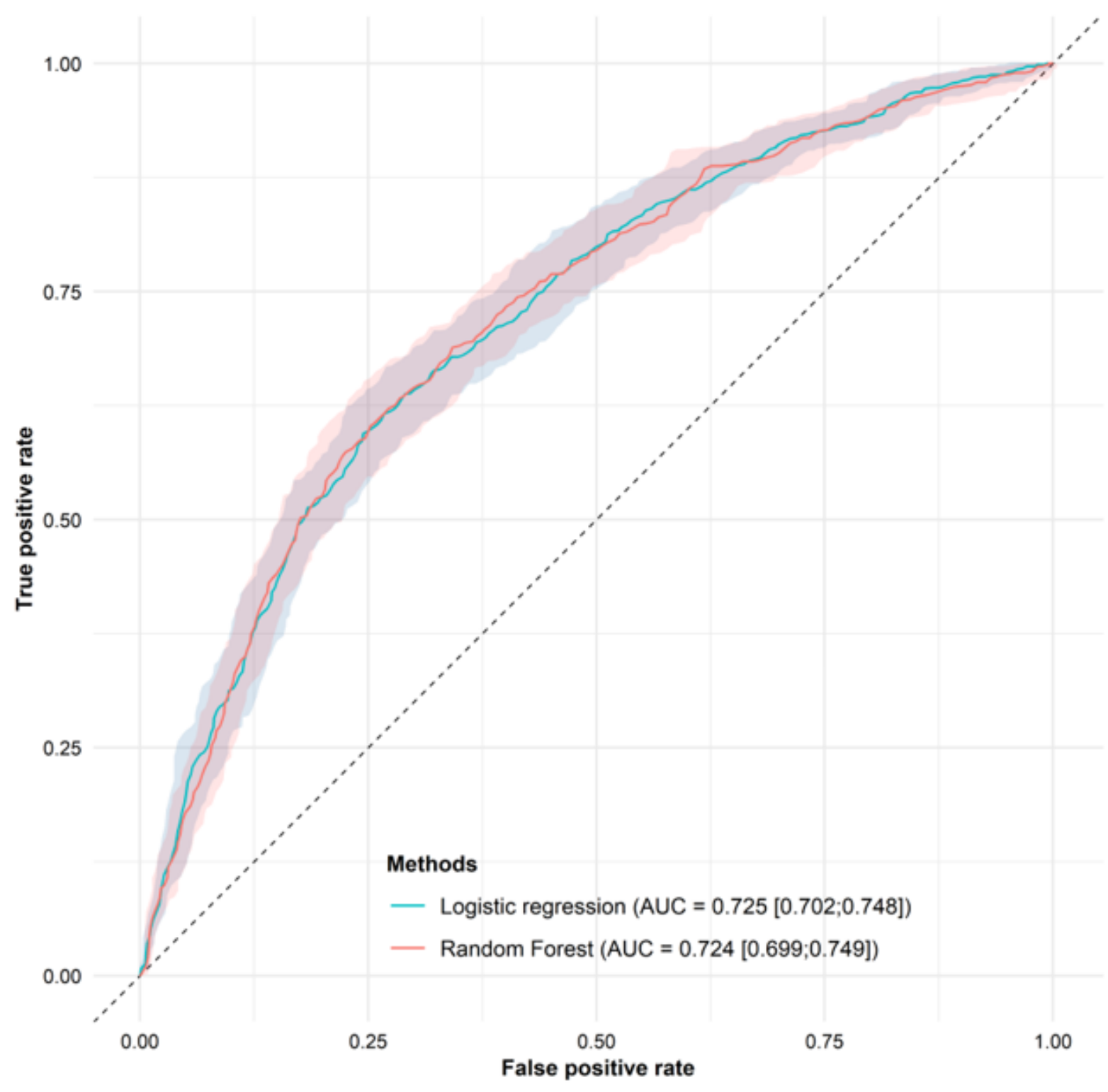

Figure 4. ROC curves comparison. 